\title{
Neurofibromin inactivation impairs osteocyte development in Nf1Prx1 and Nf1Col1 mouse models
}

\author{
Jirko Kühnisch a,b,*, Jong Seto ${ }^{\text {c,d }}$, Claudia Lange ${ }^{\text {c,e }}$, Sabine Stumpp a ${ }^{\text {a }}$ Karolina Kobus ${ }^{\text {b }}$, Julia Grohmann ${ }^{\text {b }}$, \\ Florent Elefteriou $^{\text {f }}$, Peter Fratzl ${ }^{\text {c,g }}$, Stefan Mundlos ${ }^{\text {a,b,g }}$, Mateusz Kolanczyk a,b,* \\ a Institute for Medical Genetics and Human Genetics, Charité, Universitätsmedizin Berlin, Berlin, Germany

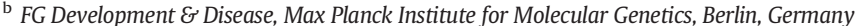 \\ c Department of Biomaterials, Max Planck Institute for Colloids and Interfaces, Potsdam, Germany \\ d Department of Chemistry, École Normale Superiéure, 24 rue Lhomond, Paris 75005, France \\ e Institut für Physiologische Chemie, MTZ, Medizinische Fakultät Carl Gustav Carus, Technische Universität Dresden, Dresden, Germany \\ ${ }^{f}$ Department of Medicine, Pharmacology and Cancer Biology, Center for Bone Biology, Vanderbilt University Medical Center, Nashville TN, USA \\ ${ }^{g}$ Berlin-Brandenburg Center for Regenerative Therapies (BCRT), Berlin, Germany
}

\section{A R T I C L E I N F O}

\section{Article history:}

Received 11 March 2014

Revised 6 June 2014

Accepted 9 June 2014

Available online 17 June 2014

Edited by: Shu Takeda

\section{Keywords:}

Bone

NF1

neurofibromin

osteocyte

MAPK

\begin{abstract}
A B S T R A C T
Neurofibromin has been identified as a critical regulator of osteoblast differentiation. Osteoblast specific inactivation of neurofibromin in mice results in a high bone mass phenotype and hyperosteoidosis. Here, we show that inactivation of the Nf1 gene also impairs osteocyte development. We analyzed cortical bone tissue in two conditional mouse models, Nf1Prx1 and Nf1Col1, for morphological and molecular effects. Backscattered electron microscopy revealed significantly enlarged osteocyte lacunae in Nf1Prx1 and Nf1Col1 mice (level E2: ctrl = $1.90 \pm$ $0.52 \%$, Nf1Prx1 $=3.40 \pm 0.95 \%$; ctrl $1.60 \pm 0.47 \%$, Nf1Col1 $2.46 \pm 0.91 \%$ ). Moreover, the osteocyte lacunae appeared misshaped in Nf1Prx1 and Nf1Col1 mice as indicated by increased Feret ratios. Strongest osteocyte and dendritic network disorganization was observed in proximity of muscle attachment sites in Nf1Prx1 humeri. In contrast to control cells, Nf1Prx1 osteocytes contained abundant cytosolic vacuoles and accumulated immature organic matrix within the perilacunar space, a phenotype reminiscent of the hyperosteoidosis shown Nf1 deficient mice. Cortical bone lysates further revealed approx. twofold upregulated MAPK signalling in osteocytes of Nf1Prx1 mice. This was associated with transcriptional downregulation of collagens and genes involved in mechanical sensing in Nf1Prx1 and Nf1Col1 bone tissue. In contrast, matrix gla protein (MGP), phosphate regulating endopeptidase homolog, $\mathrm{X}$-linked (PHEX), and genes involved in lipid metabolism were upregulated. In line with previously described hyperactivation of $N f 1$ deficient osteoblasts, systemic plasma levels of the bone formation markers osteocalcin (OCN) and procollagen typ I N-propeptide (PINP) were approx. twofold increased in Nf1Prx1 mice. Histochemical and molecular analysis ascertained that osteocytes in Nf1Prx1 cortical bone were viable and did not undergo apoptosis or autophagy. We conclude that loss of neurofibromin is not only critical for osteoblasts but also hinders normal osteocyte development. These findings expand the effect of neurofibromin onto yet another cell type where it is likely involved in the regulation of mechanical sensing, bone matrix composition and mechanical resistance of bone tissue.
\end{abstract}

(c) 2014 Elsevier Inc. All rights reserved.

\section{Introduction}

Osteocytes constitute $90 \%$ of all cells of adult cortical bone [1]. Embedded in the mineralized matrix, osteocytes communicate with one another through a network of dendritic processes, the canalicular network, and are capable of regulating local bone turnover by recruiting osteoclasts and osteoblasts [1-3]. Osteocytes are formed by differentiation of mesenchymal progenitor cells into osteoblasts and their subsequent

\footnotetext{
* Corresponding authors at: Institute for Medical Genetics and Human Genetics, Charité Berlin - Campus Virchow, Augustenburger Platz 1, 13353 Berlin, Germany.

E-mail addresses: jirko.kuehnisch@gmx.de (J. Kühnisch), kolanshy@molgen.mpg.de (M. Kolanczyk).
}

incorporation into the bone matrix [1]. During differentiation osteocytes acquire specific morphological properties including dendritic processes and small cell body volume. This specific adaptation of morphology and ultrastructure allows osteocytes to perform their function as mechanosensory cell embedded within the mineralized bone. The osteocyte canalicular system is thought to facilitate the mechanosensory function by providing attachment sites for the cell processes, which are the main sites of the mechanical stimulation [4].

Osteocytes respond to mechanical stimulation with rapid synthesis of nitric oxide (NO) and prostaglandins [1]. This in turn leads to downregulation of WNT pathway inhibitors SOST and DKK1, which constitutes a bone-anabolic signal [5]. Osteocytes actively remodel their perilacunar matrix with help of metalloproteinases thus regulating 
phosphate and calcium availability [6]. Both osteoblasts as well as the early osteocytes regulate the degree of matrix mineralization in their surrounding with help of SIBLING family of proteins: osteopontin (OPN), dentin matrix protein 1 (DMP1) and matrix extracellular phosphoglycoprotein (MEPE) as well as with Matrix Gla Protein (MGP) [7]. While OPN, MEPE and MGP inhibit mineralization, DMP1 is a promineralization factor [8]. Inactivating mutations of DMP1 or endopeptidase PHEX result in bone mineralization defects that are associated with increased FGF23 expression in osteocytes [9]. FGF23 is an endocrine regulator of systemic phosphate metabolism that is mainly synthesized by osteocytes [8]. Mechanical stimulation ensures osteocyte viability by preventing apoptosis [10]. This is achieved at least in part by the activation of focal adhesion kinases (FAKs) and extracellular signals-regulated kinases (ERKs) [10]. In addition, ERK signalling is critical for Dmp1 expression and osteocyte differentiation [11]. Apart from apoptosis osteocytes can undergo a process aimed at self-preservation called autophagy, which is induced by glucocorticoids [12].

Biallelic inactivation of the $N f 1$ gene results in a profound skeletal pathology in patients with Neurofibromatosis type 1 (NF1) and corresponding conditional mouse models [13-15]. Inactivation of Nf1 specifically in osteoblasts (Nf1Col1 mice) causes increased collagen synthesis, but inhibits bone mineralization resulting in osteoidosis [13,14]. Osteoblast dysfunction is therefore an important factor contributing deterioration of bone material properties [13]. We have shown that ablation of Nf1 in undifferentiated limb mesenchyme and derivative tissues produces in Nf1Prx1 mice a severe bone phenotype characterized by increase of micro-porosity, hypomineralization, a generalized defect of organic matrix formation, and persistence of ectopic blood vessels that are associated with localized macro-porotic bone lesions [16]. This phenotype overlaps with findings made in NF1 patient material suggesting a complex mechanism leading to bone fragility in NF1 [16]. Increased micro-porosity and reduced organic matrix quality are likely caused by defective osteocyte development. In order to further characterize the role of osteocytes in NF1 bone dysplasia, we now focused on the analysis of molecular and histological aspects of the pathology in Nf1 deficient murine cortical bone. We hypothesize that Nf1 affects osteocyte differentiation.

\section{Methods}

Mouse breeding and genotyping

Nf1Prx1 and Nf1Col1 mice were bred and genotyped as described previously $[13,14,17]$. All experimental procedures were approved by the 'Landesamt für Gesundheitsschutz und Technische Sicherheit (LaGeTSi), Berlin, Germany (protocol number ZH 120) and the Institutional Animal Care and Use Committee (IACUC) at the Vanderbilt University Medical Center (protocol number M/06/508).

\section{Protein and mRNA analysis}

Cortical bone was mechanically cleaned from adjoining muscle and connective tissue. Bone marrow was removed by a syringe flush. Separated cortical bone tissue was crashed after liquid nitrogen incubation, mRNA lysates were generated with Trizol reagent (Invitrogen, Carlsbad, USA) and protein lysates were obtained by incubation with RIPA buffer. Protein expression analysis was performed with Western blot after SDSpolyacrylamide electrophoresis and transfer onto PVDF (Amersham, USA) membrane. For Western blot analysis, membranes were incubated with the following antibodies: anti-activeRAS \#26909 (Neweastbio), anti-RAS \#14022 (SantaCruz), anti-phospho-p42/44 (pERK1/2) \#9102 (Cell Signaling), anti-p44 (ERK1) \#4372 (Cell Signaling), anti-pAMPK \#4370S (Cell Signaling), anti-AMPK \#9197 (Cell Signaling), antipMEK1/2 \#9121S (Cell Signaling), anti-MEK1/2 (Cell Signaling), Sost (R\&D, AF1589), PARP \#9542 (Cell Signaling), cleaved PARP \#9541 (Cell Signaling), LC3AI/II (D50G8), LIMP II \#25867 (Santa Cruz), and
anti-GAPDH \#Sc-25778 (Santa Cruz). For mRNA expression analysis full RNA was isolated according to standard protocols and transcribed into cDNA with SuperscriptII (Invitrogen, Carlsbad, USA). Expression analysis was performed with a Taqman 7500 (ABI, USA) using Gapdh as endogenous control. The following primer were used (5'-3'orientation): Mepe_f_TGTCTGTTGGACTGCTCCTC, Mepe_r_tGTGAATGCTGTCTTGATT GC; Sost_f_AACAACCAGACCATGAACCG, Sost_r_GTCAGGAAGCGGGTG TAGTG; Dmp1_f_TGTCATTCTCCTTGTGTTCTTTTG, Dmp1_r_TCTTCAGAG CTTTCAGATTCAGTATTG; Phex_f_CTGGTGTTGGGCACACTG, Phex_r_CG GCTTCTATGCATTCTGG; MGP_f_ACAGGAGAAATGCCAACACC, MGP_R_ GTAGTCATCGCAGGCCTCTC; E11_f_GAGGCTCCAACGAGATCAAG, E11_r_ TCCAGTAGCACCTGTGGTTG; Gapdh_f_AACTTTGGCATTGTGGAAGG, Gapdh_r_TCTTCTGGGTGGCAGTGATG.

\section{Microarray hybridization and differential expression evaluation}

RNA was isolated from cortical bone using peqGOLD TriFast (PeqLab Biotechnologie $\mathrm{GmbH}$ ) according to the supplied protocol. The cRNA synthesis and microarray hybridizaton was done according to standard protocols applied in the Laboratory of Functional Genome Research Charite - Core Facility. A total of 8 high density oligonucleotide mouse GENE 1.0 ST arrays (Affymetrix) were used in this study. Samples from each animal ( $N f 1$, and 4 wild-type) were applied individually to each array. All the procedures and hybridization were performed according to the Genechip expression technical manual (Affymetrix). One way ANOVA analysis was used to reveal the genes significantly deregulated in the Nf1 deficient muscles ( $F$ factor $\geq 20$ ).

\section{Histology}

For histological analysis, adult limbs were fixed in buffered $4 \%$ PFA, dehydrated in subsequent ethanol steps and embedded in methyl methacrylate (MMA) according standard laboratory procedures [18]. For histological assessment $5 \mu \mathrm{m}$ plastic sections (RM2255, Leica, Germany) were stained with a combined von Kossa/Toluidin procedure $[14,19]$. Bright light microscopy occurred with an Olympus BX60 (Olympus, Japan). To analyse presence of acid bone matrix proteins sections were stained with AgNOR [20]. Collagenous bone matrix was assessed with bright light microscopy after Toluidin and Safranin O staining [21] (Leica DMRB, Germany). Histomorphometric analysis was performed with AxioVision Software (Zeiss, Germany). Nomenclature was used according ASBMR suggestions [22]. Statistical analysis was performed with unpaired t-test, ${ }^{*} \mathrm{p} \leq 0.05,{ }^{* *} \mathrm{p} \leq 0.01$.

\section{Immunohistology}

Immunodetection of proteins within mineralized cortical bone was achieved according to a standard procedure [23]. Briefly, frozen tissue was sectioned $(5 \mu \mathrm{m})$ on a cryostat (Leica CM3050 S, Wetzlar, Germany). Subsequently, sections were fixed in PBS buffered $4 \%$ PFA, permeabilized with $0.1 \%$ saponin in PBS buffered $3 \%$ BSA, blocked with $5 \%$ donkey serum, and antibodies were incubated in PBS buffered $3 \%$ BSA. The following antibodies were used for immunodetection: panendothelial cell antigen (550563, BD Pharmingen, USA), DMP1 (AF4386, R\&D, USA), anti-rabbitHRP (\#7074, Cell Signalling, USA), anti-ratAlexa555 (A11081, Invitrogen, USA), and anti-sheepAlexa555 (A21436, Invitrogen, USA). Sections were analyzed with confocal microscopy (lsm 510, Zeiss, Germany). Isotype specific secondary antibodies were used in negative control experiments (data not shown).

\section{Biochemical analysis}

Plasma was collected after decapitation with standard protocols utilizing Li-Heparin capillary tubes (GK $150200 \mu \mathrm{l} \mathrm{Gel} \mathrm{grün,} \mathrm{Kabe,}$ Germany). Biochemical analysis of bone parameters was achieved with enzyme linked immunoassays detecting osteocalcin (EIA-4010, 
DRG Instruments GmbH, Germany), Rankl (MTR00, R\&D Systems, USA), Opg (MOP00, R\&D Systems, USA), procollagen I N-terminal propetide (PINP) (E90957Mu, Uscn, China), Fgf23 (Kainos, Japan), and Mepe (E91232Mu, Uscn, China). Serum and urine analysis of phosphate, calcium and creatinin was measured at the clinical laboratory core facility according standard procedures.

Scanning electron microscopy (SEM) and backscattered electron microscopy (BSE)

For Ot. morphology assessment, MMA embedded humeri were cut either along the cross or longitudinal sectional direction of the main bone axis and analyzed by SEM and BSE respectively.

For SEM analysis, humeri were trimmed with a hard tissue microtome to the appropriated height levels (E1, E2, E3). Etching occurred with phosphorous acid ( $37 \%, 12 \mathrm{sec}$ ), wash three times $\mathrm{H} 2 \mathrm{O}$, sodium hypochlorite ( $4 \%, 5 \mathrm{~min}$ ), and wash for $5 \mathrm{~min}$ with $\mathrm{H} 2 \mathrm{O}$. Subsequently, samples were spattered with gold/palladium (coating system MED020 BAL-TECH) and imaged with a SEM (DSM982GEMINI, Zeiss, Germany).

For BSE imaging MMA embedded humeri were sectioned to a medial position showing both cortices and the bone marrow cavity. To investigate bones with ESEM (FEI-Company, Oregon, USA) plastic embedded humeri were polished to mirror finish to avoid a rough and scratched sample surface. Therefore, different grades of abrasive paper have been applied for several minutes. Polished samples were investigated in low vacuum ( 0.75 Torr) using BSE mode at a working distance of $10 \mathrm{~mm}$. The electron beam energy was set to $10 \mathrm{kV}$. A solid state detector (SSD) was used to measure the BSE signal from the sample surface. The resulting grey level images of the mineralized bone tissue reveal regions of high calcium content in bright and regions of low calcium content in dark grey, representing high and low mineral concentration in the bone sample, respectively [24].

\section{Results}

\section{Altered osteocyte morphology in Nf1Prx1 and Nf1Col1 humerus cortex}

Nf1 was inactivated in the mesenchymal lineage (Nf1Prx1) and in pre-osteoblasts (Nf1Col1) as previously described [16]. In order to analyse the morphology of osteocyte (Ot.) lacunae, we performed backscattered electron microscopy (BSE) on methacrylate embedded humeri of three month old mice. Cortical bone sections were analysed along the proximo-distal axis. Three regions of interest (ROI E1-E3) were selected for qualitative and quantitative analysis. BSE revealed striking qualitative changes in Ot. lacunae shape in Nf1Prx1 and to a lesser degree in Nf1Col1 bone samples (Figs. 1A, B). In controls, Ot. lacunae were spindle-shaped and aligned longitudinally towards the long bone axis in all ROIs (Figs. 1A, B). However, in Nf1Prx1 bones lacunae appeared enlarged and irregularly distributed (Fig. 1A). Similar but less pronounce changes were apparent in Nf1Col1 bones (Fig. 1B). Quantitative analysis revealed increase of the relative Ot. lacunae area per bone area (Ot.Ar/B.Ar) in mutants as compared to respective controls (level E2: ctrl $=1.90 \pm 0.52 \%$, Nf1Prx1 $=3.40 \pm 0.95 \%$; ctrl $1.60 \pm 0.47 \%$, Nf1Col1 $2.46 \pm 0.91 \%$ ) (Fig. 1C, Tables S1-2). In contrast, Ot. number was not increased, with the exception of Ot. number in ROI E2 in Nf1Prx1 mice (Fig. 1D). Lacunae shape was assessed by measuring lacunae area (Ot.Ar) and Feret ratio on BSE images (Feret value $0=$ line; $1=$ circle) (Fig. 1E). Ot. area and Feret ratio was increased in Nf1Prx1 and Nf1Col1 cortical bone samples (Fig. 1E), indicating a general enlargement as well as more spherical shape of the Ot. in mutant bones.

Changes in osteocyte canalicular network and cellular morphology in Nf1 deficient bones

BSE microscopy revealed a more spherical shape of Ot. in Nf1 deficient bone tissue (Fig. 2A). In order to characterize changes of Ot. dendrite network morphology we applied complementary histological techniques. AgNOR silver staining labels acidic bone matrix proteins, allowing for visualization of the entire Ot. canalicular network (Fig. 2B) [20]. In control bone tissue, the most homogenous distribution of canalicular network was observed in the region E2, which is far from the muscle attachment sites. In this region Ot. were aligned with their long axis towards the circular collagen orientation. Control Ot. had long fragments of canaliculi visible in the section plane perpendicularly to the cell body (Fig. 2B). In contrast, canaliculi in Nf1Prx1 bone appeared shorter and unevenly distributed in proximity of mutant Ot., indicating either altered dendrite number or distribution (Fig. 2B). Striking changes of the Ot. morphology were also seen by scanning electron microscopy (SEM) (Figs. 2C and 3). Analysis of subcellular organization by transmission electron microscopy (TEM) revealed unusually abundant and large vacuoles within the cytosol of Nf1Prx1 Ot. (Fig. 2D). Many osteocytes also showed presence of immature collagen in the perilacunar space.

Anatomical variation in the extent of osteocyte and canalicular network changes in Nf1 deficient bone

Analysis of the SEM micrographs revealed that morphological changes of the Nf1Prx1 Ot. dendritic network were most striking in the areas proximal to muscle attachment sites (Figs. 3A-B). Particularly in the proximity of tuberositas tibiae and tuberositas medialis in the region E1 and E3 Ot. appeared misshaped and the canalicular network disturbed. In contrast, much less severe were changes of Ot. morphology in the area E2 which is located in the mid shaft bone. These data indicate that $N f 1$ deficient Ot. in the cortical bone show morphological changes predominantly in the areas exposed to high mechanical load.

\section{Osteocyte viability is not impaired in Nf1Prx1 bone}

In order to check if Nf1 deficiency might be associated with Ot. apoptosis or autophagy, we performed histological and molecular marker analyses. We did not detect empty Ot. lacunae within Nf1Prx1 bone cortices, which would be indicative of Ot. death (Fig. 4A). In line with this, no TUNEL positive cells were detected within cortical bone (Fig. 4B) and the levels of cleaved-PARP in cortical bone lysates were normal (Fig. 4C). Levels of autophagy marker expression LC3AII and LC2BII were also unaffected (Fig. 4D). Collectively, these data indicate that Ot. viability is not impaired by Nf1 inactivation in bone.

\section{Dysregulation of a subset of mineralization regulating genes in Nf1 deficient cortical bone}

Neurofibromin critically regulates Ras-MAPK signalling [25]. In line with this implication, we detected increased activation of Ras, MEK1 and ERK1/2 in cortical bone lysates of Nf1Prx1 mice (Fig. 5A). Additionally, we found increased activation of AMP activated protein kinase (AMPK), which is a marker of ongoing energetic stress (Fig. 5A) [26]. Neither serum level of matrix extracellular phosphoglycoprotein (Mepe) nor its mRNA transcript level was significantly deregulated (Fig. 5B). Similarly, expression levels of Dmp1, E11/podoplanin, and Sost were not altered in cortical bone of Nf1Prx1 mice as compared to controls (Fig. 5B). However, expression of the Mgp gene, an osteoblast and vessel expressed mineralization inhibitor, was significantly increased (Fig. 5B). Additionally, expression of Phex, known to be mutated in X-linked hypophosphatemic rickets, was also increased [27]. Systemic levels of osteoprotegerin (Opg) and receptor activator of NF- $\kappa$ B ligand (Rankl) were unaffected suggesting normal osteoblast-osteoclast coupling (Fig. 5C). Similarly, normal level of the Ot. specific bone mineral regulator Fgf23 indicate no systemic defect of mineral homeostasis. Consistent with previous observations in Nf1Col1 mice [13], plasma levels of osteocalcin (Ocn) and procollagen I N-terminal propeptide (PINP), biomarkers of osteoblast activity, were approx. 2-fold increased 
A

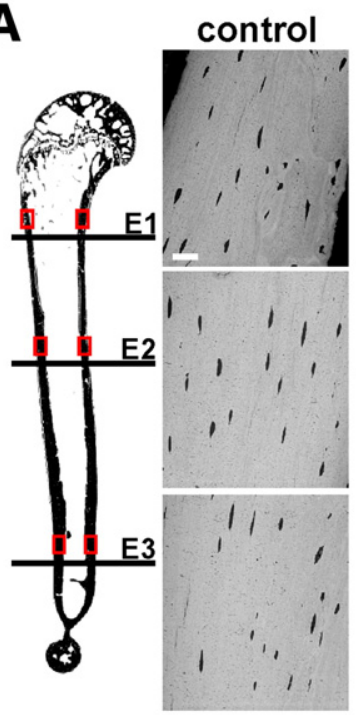

C

C BSE 2D area measurement

- control $=\mathrm{Nf1}$ Col1 $=$ control $\| \mathrm{Nf1}$ Prx1

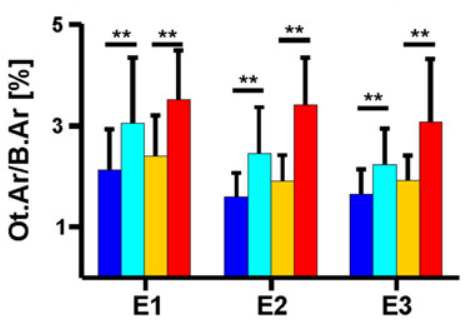

$\mathrm{Nf}^{\mathrm{Prx1}} \quad$ B

B control
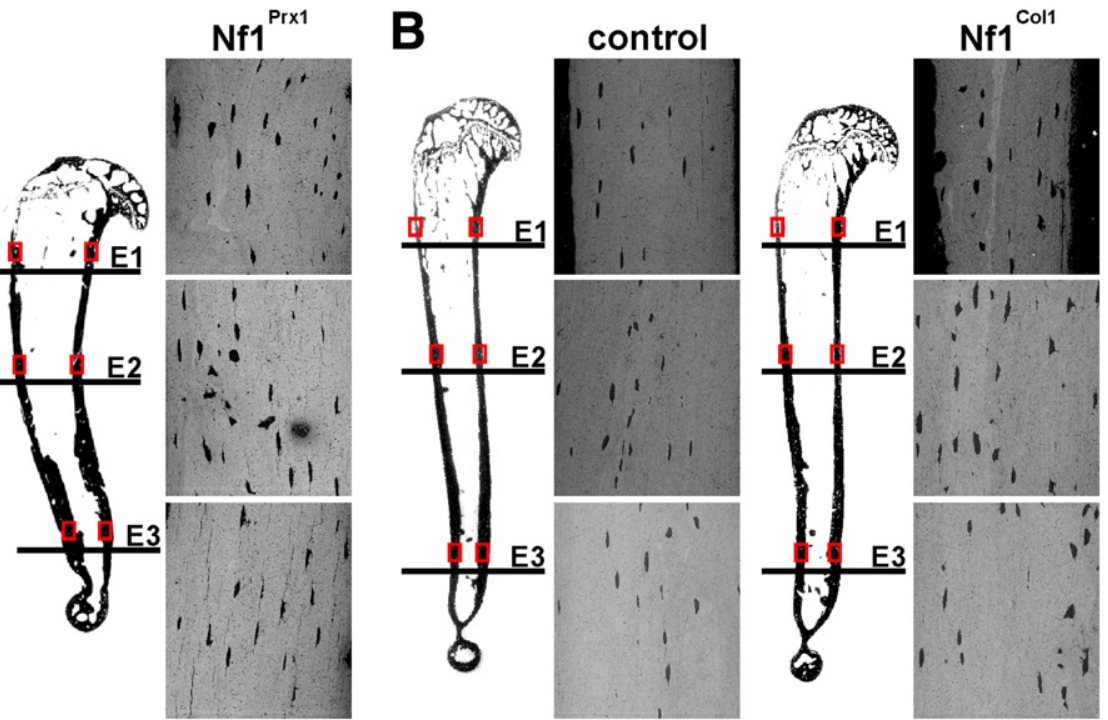

D.

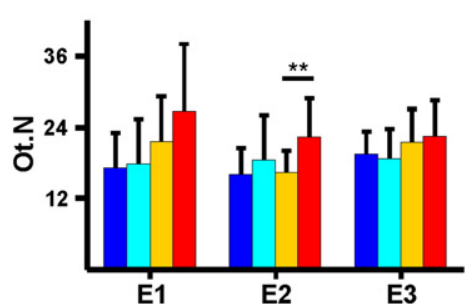

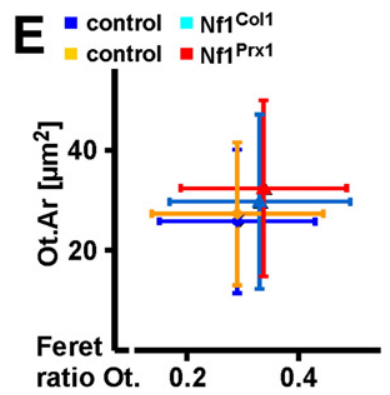

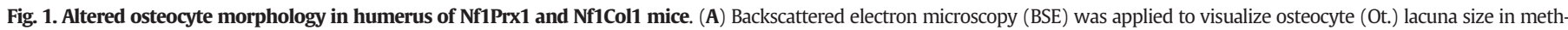

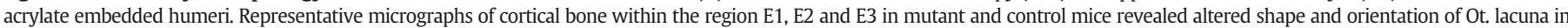

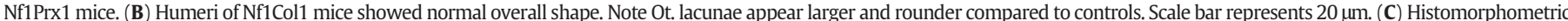

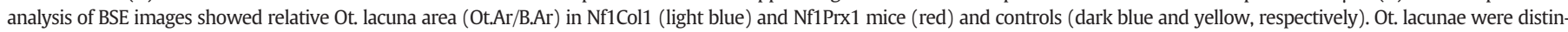

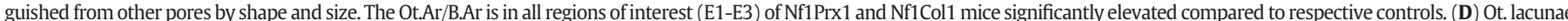

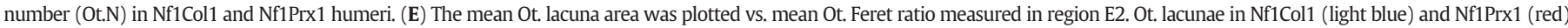

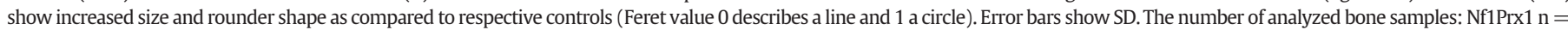
5 , controls $n=5$; Nf1Col1 $n=3$, controls $n=3$. Statistical analysis was performed with unpaired Student t-test, ${ }^{*} p \leq 0.05{ }^{* *} p \leq 0.01$.

A

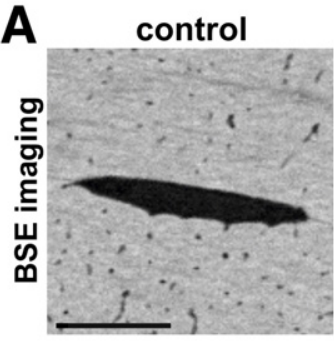

C

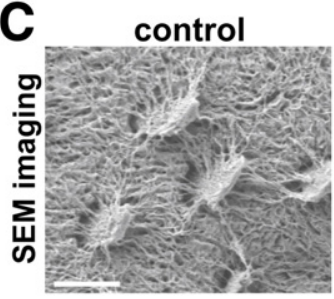

$N f 1^{\text {Prx } 1}$
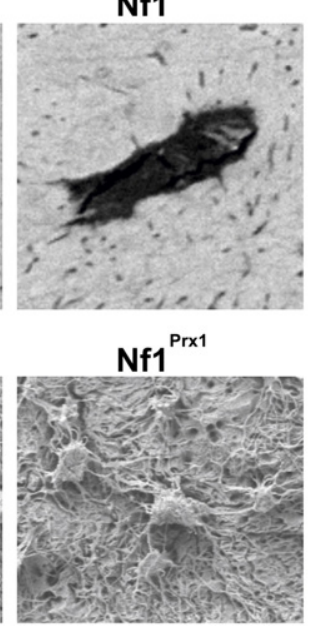

B

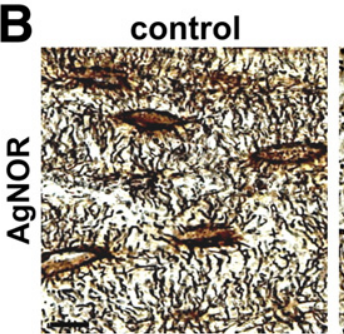

D

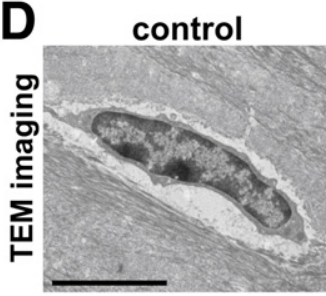

$N f 1^{\text {Prin }}$

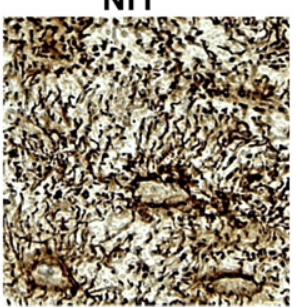

Nf1 ${ }^{\text {Prx1 }}$

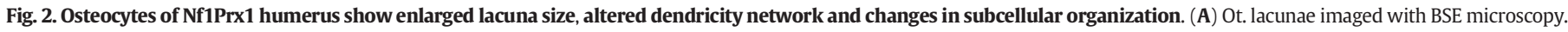

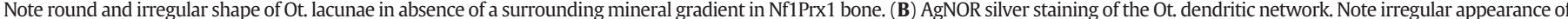

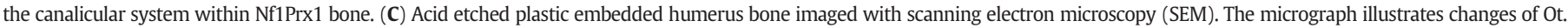

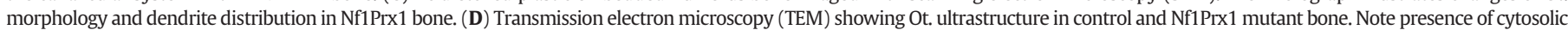
vacuoles and amorphous collagen in the perilacunar space of Nf1Prx1 bone. Scale bars are $10 \mu \mathrm{m}$. 
A

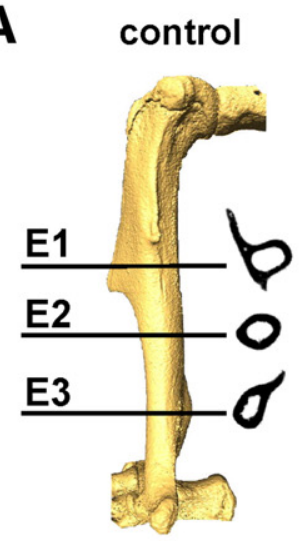

B

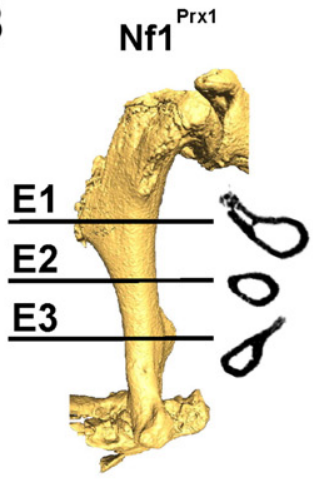

E1a
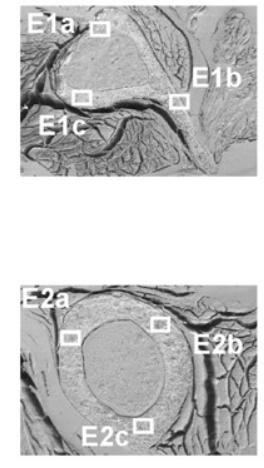

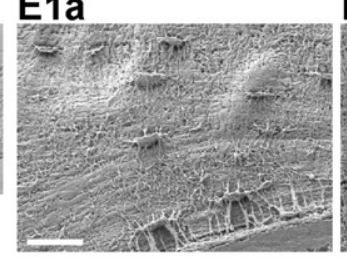

\section{E2a}
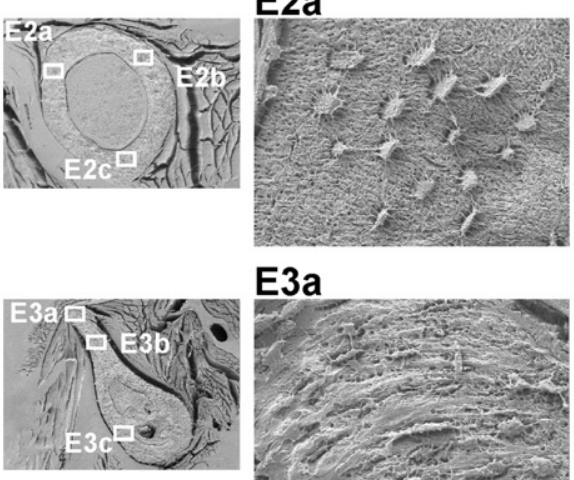

\section{E3a}

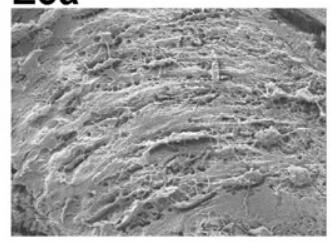

E1a
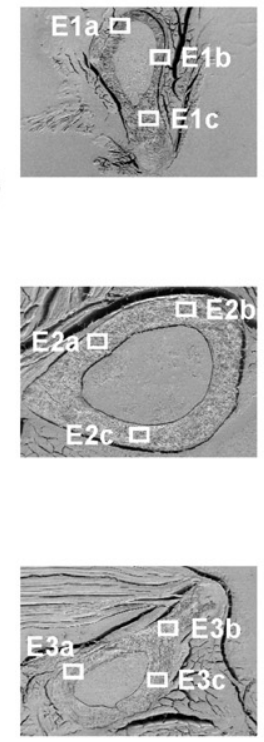

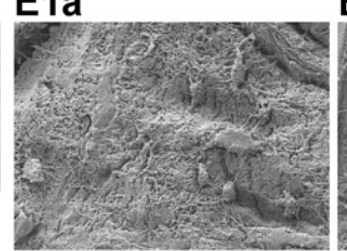

\section{E2a}

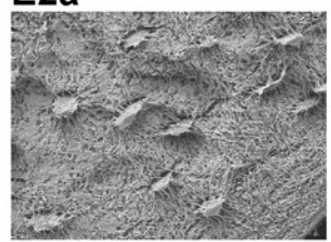

E3a

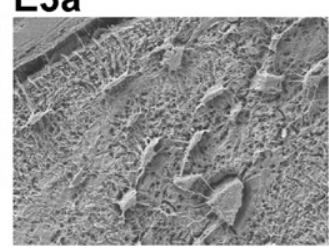

E1b

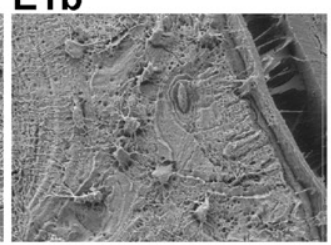

E2b

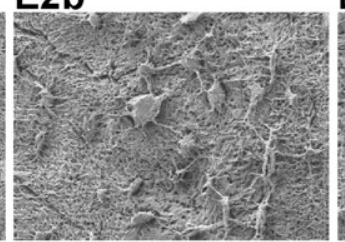

E3b

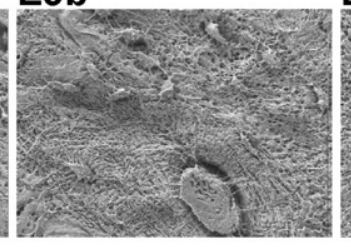

E1b

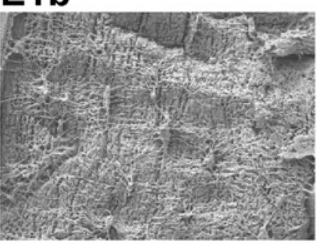

E2b

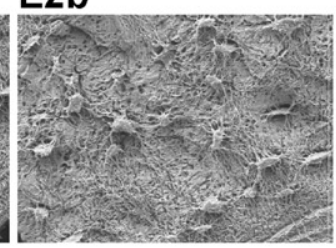

E3b

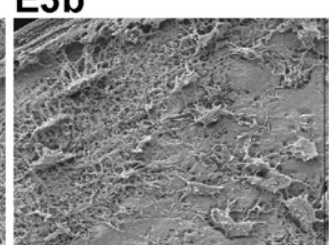

E1c

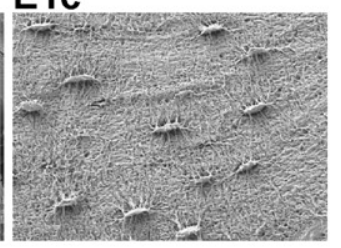

E2c

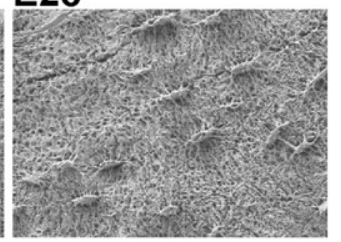

E3c

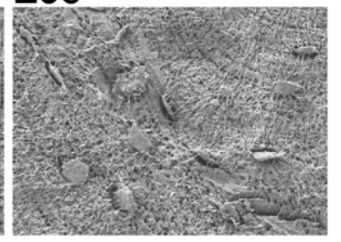

E1c

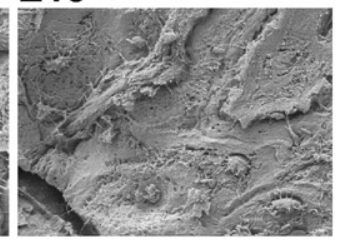

E2C

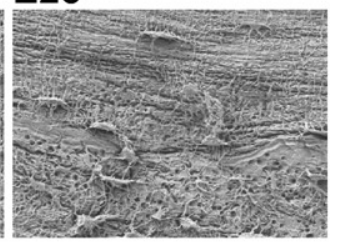

E3c

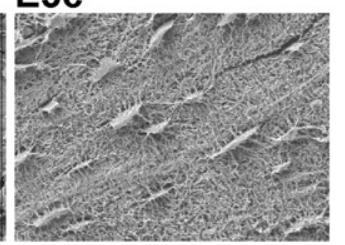

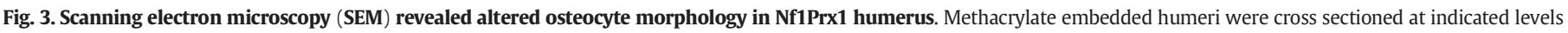

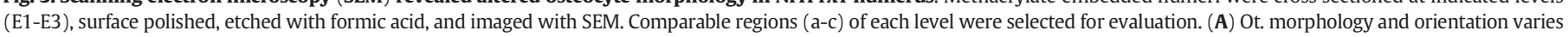

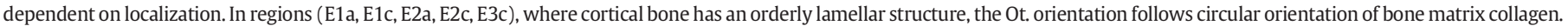

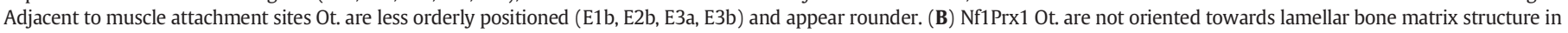

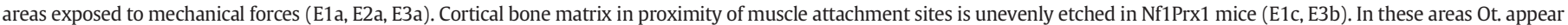
irregularly distributed and have altered shaped. Scale bar represents $20 \mu \mathrm{m}$.

(Fig. 5C). Altogether, these results show a selective effect of Nf1 inactivation on the expression of a subgroup of genes involved in matrix mineralization, but no global derangement of the Ot. homeostatic gene network: Sost, Fgf23 and Dmp1.

Transcriptional upregulation of genes involved in energy derivation/storage and downregulation of ECM synthesis marks energetic stress within Nf1 deficient cortical bone

To gain more information about the molecular aspects of the observed phenotype, we analysed transcriptional profiles of Nf1 deficient bones using Affymetrix Mouse Gene 1.0ST microarrays. Gene expression profiling was done for four mutant and four control animals, and the significant gene regulations determined with one-way analysis of variance (ANOVA). We detected 565 significantly upregulated and 535 downregulated uniquely identified (DAVID-ID) genes in Nf1Col1 cortical bone samples (cut-off: $\mathrm{p} \leq 0.01$ ) and 672 significantly upregulated and 235 downregulated transcripts (cut-off: $\mathrm{p} \leq 0.02$ ) in Nf1Prx1 cortical bone samples (Tables S3-8). These significantly regulated genes were subjected to classification and gene class enrichment analysis [28]. Common functional groups among upregulated genes in both models were: GO:0017076 purine nucleotide binding Nf1Col1 - 88 
A $\quad$ P60

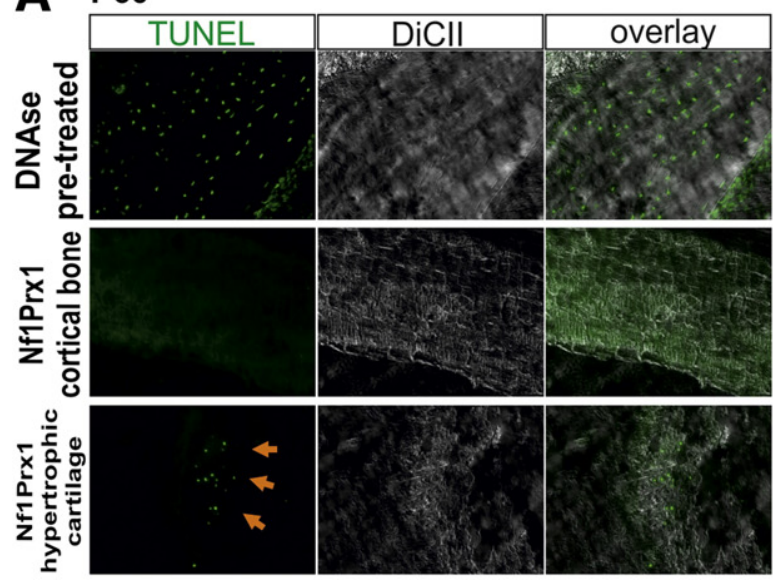

B cleaved PARP/PARP
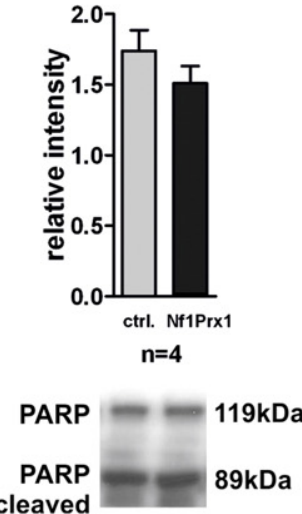

C

LC3AII/LC3AI

LC3BII/LC3BI

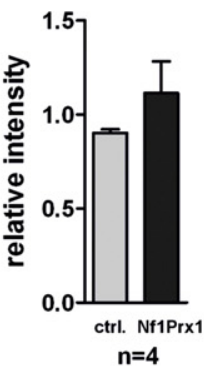

LC3AI

LC3All

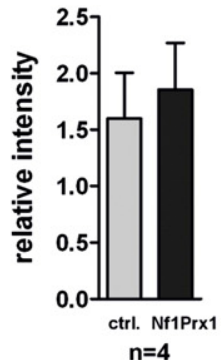

LC3BI LC3BII

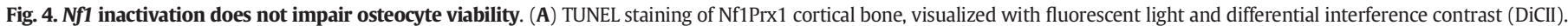

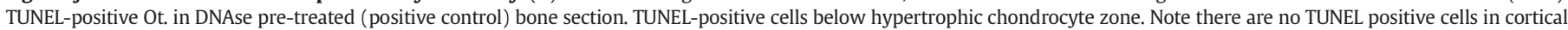

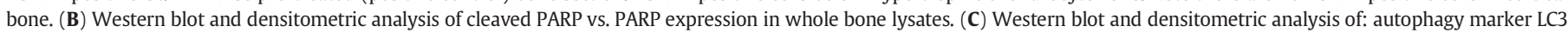
(microtubule-associated protein1 light chain 3). Normal autophagy index - band intensity ratio of intact LC3 (LC3I) vs. activated LC3 (LC3II) in Nf1Prx1 bone lysates.

genes $/ \mathrm{p}=8.09^{-7}$; Nf1Prx1- 107 genes, $\left.\mathrm{p}=7.77^{-8}\right)$; GO:0008610 $\sim$ lipid biosynthetic process (Nf1Col1 - 17 genes/p $=0.007$; Nf1Prx $1-13$ genes $/ \mathrm{p}=0.28) ; \mathrm{GO}: 0006897 \sim$ endocytosis $(\mathrm{Nf} 1 \mathrm{Col} 1-6$ genes $/ \mathrm{p}=$
0.61; Nf1Prx1- 16 genes/p = 0.002); G0:0005764 lysosome or GO:0000323 lytic vacuole (Nf1Col1 - 6 genes/p = 0.58; Nf1Prx115 genes/p = 0.005) (Fig. 5D) (Tables S4, S8).

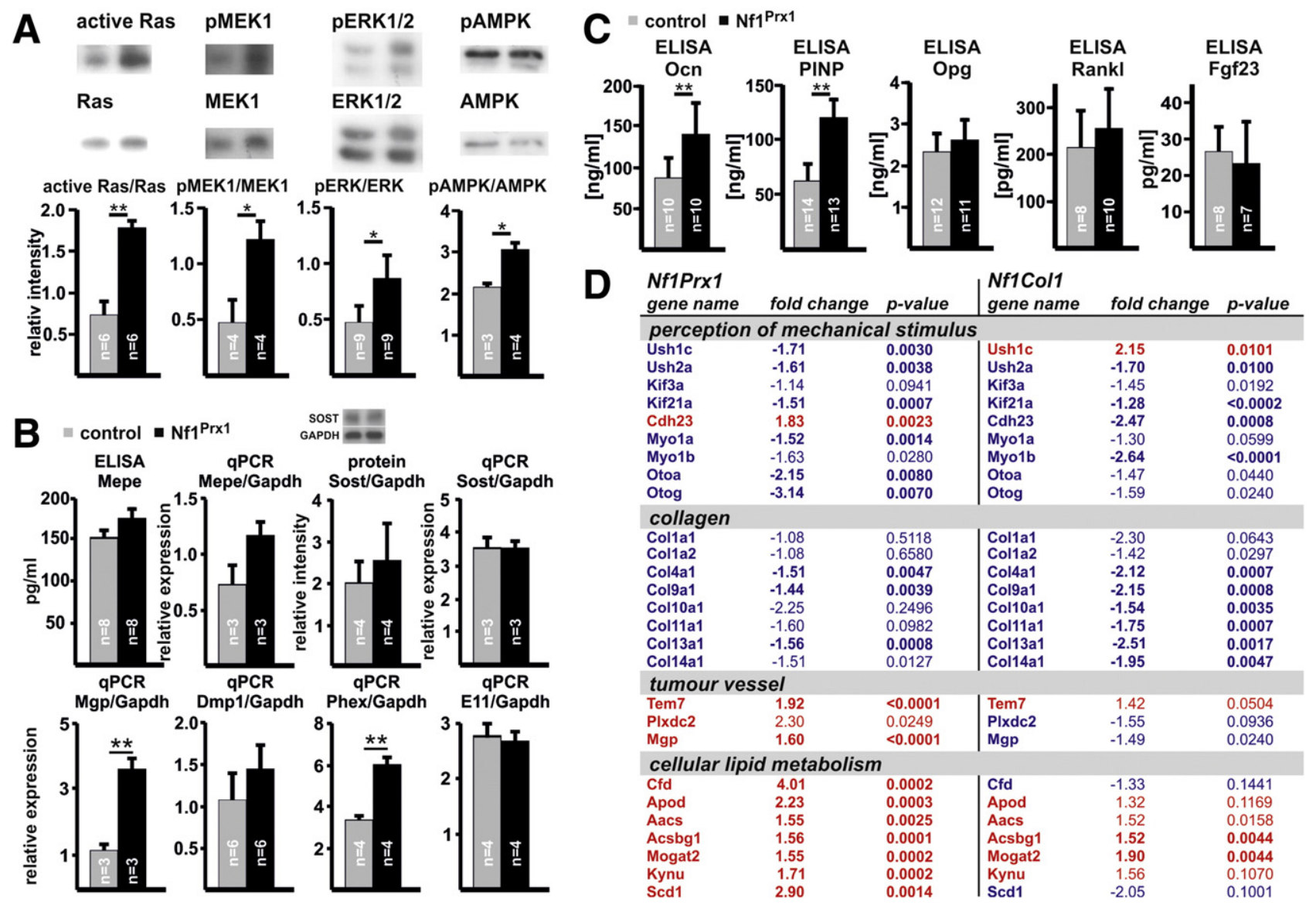

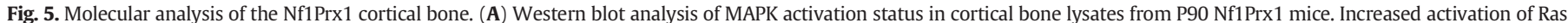

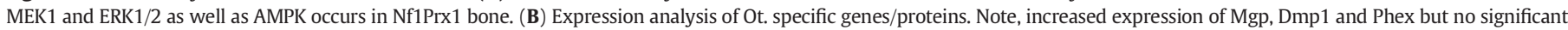

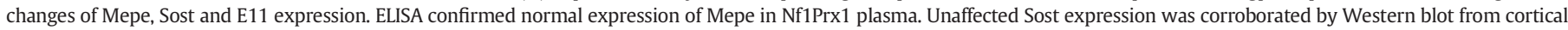

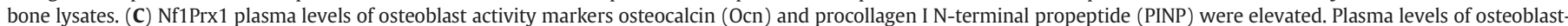

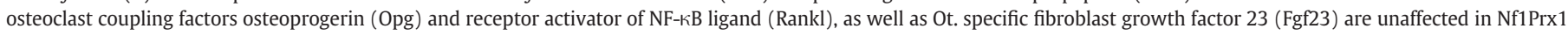

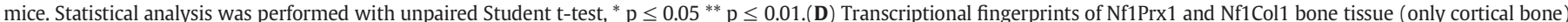

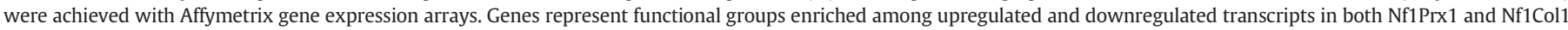

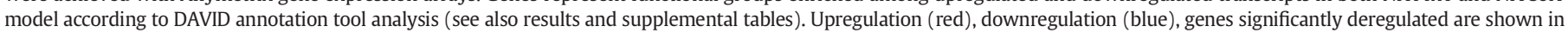
bold. P-values were determined with one-way ANOVA analysis. 
In both mouse models this was paralleled by downregulation of genes of the following GO categories: GO:0031012 extracellular matrix (Nf1Col1 - 62 genes, $\mathrm{p}=1.96^{-32}$; Nf1Prx1 - 14 genes, $\mathrm{p}=7.13^{-5}$ ); GO:0005581 collagen (Nf1Col1 -8 genes, $\mathrm{p}=9.25^{-7}$; Nf1Prx1 -9 genes, $\left.\mathrm{p}=5.44^{-6}\right)$; GO:0015629 $\sim$ actin cytoskeleton (Nf1Col1 - 19 genes, $\mathrm{p}=6.59^{-5}$; Nf1Prx1- 13 genes, $\left.\mathrm{p}=5.27^{-6}\right) ; \mathrm{GO}: 0007155 \sim$ cell adhesion (Nf1Col1 - 63, $\mathrm{p}=8.84^{-22}$; Nf1Prx1- 17 genes, $\mathrm{p}=5.06^{-4}$ ); GO:0050954 sensory perception of mechanical stimulus (Nf1Col1 - 5 genes, $\mathrm{p}=0.24$; Nf1Prx1- 6 genes, $\mathrm{p}=0.003)$; GO:0048839 $\sim$ inner ear development (Nf1Col1 - 8 genes, $p=0.009$; Nf1Prx1- 4 genes, $\mathrm{p}=0.07$ ); GO:0003774 motor activity (Nf1Col1 - 14 genes, $\mathrm{p}=1.09^{-4}$; Nf1Prx1- 11 genes, $\mathrm{p}=4.03^{-6}$ ) (Table S5, S7). Additionally, in both models some genes belonging to the GO:0050954 sensory perception of mechanical stimulus were upregulated (e.g. Myo7a and Cdh23). Moreover, the endothelial marker 7 (Tem7/Plxdc1) that is highly expressed in osteosarcoma cells was upregulated in Nf1Prx1 and Nf1Col1 bones (Fig. 5D). Thus, transcriptional profiles of Nf1Prx1 and Nf1Col1 bones share increased expression of genes involved in nutrient supply, energy derivation and energy storage. Commonly down-regulated genes are involved in matrix synthesis as well as in cytoskeleton organization. Dysregulated expression of genes known to be crucially involved in mechanosensory cilia function (e.g. Ush and Kif genes) points to derangement of the osteocytic mechanosensory system in Nf1 deficient bone.

\section{Discussion}

Deregulation of osteoblast and osteoclast activity in Nf1 deficient bone yield a high-turnover phenotype, reduced bone mass and low bone mineral content $[13,14,29,30]$. Detailed structural analysis revealed increased micro-porosity in Nf1Col1 and Nf1Prx1 cortical bones, which was due to increased osteocyte lacunae size but not osteocyte number [16]. However, no histological or molecular data were available to support the role of osteocytes in the NF1 bone pathology. Here, we show that osteocyte lacunae are not only enlarged but also misshaped (more spherical) upon loss of neurofibromin. Additionally, the canalicular network appears disorganized especially in proximity of muscle attachment sites. The altered shape of osteocyte lacunae and subcellular organization point towards perturbation of the cytoskeleton as main determinant of cellular morphology. This conclusion is likely as neurofibromin was recently shown to control two signalling pathways responsible for regulation of actin cytoskeleton: Rho-ROCK-LIMK2Cofilin [31], and Rac1-Pak1-LIMK1-Cofilin [32]. In this study we detected increased activity of canonical MAPK signalling in cortical bone lysates. We also showed enhanced phosphorylation of AMPK, which is activated by decreasing concentrations of adenosine triphosphate (ATP) and increasing AMP/ADP concentrations thus being an indicator of an ongoing energetic stress [33]. In line with the energetic stress hypothesis, we detected in $N f 1$ deficient bones increased expression of genes encoding purin-binding proteins and lysosomal proteins involved in energy derivation. In both Nf1Col and Nf1Prx1 bone tissue, this was further complemented by an increased expression of lipid metabolism genes suggesting that Nf1 deficient bone cells undergo metabolic rearrangement. Additionally, downregulation of multiple genes involved in the mechanosensory apparatus and cytoskeletal function suggest that the cortical bone mechanosensory function is also affected by Nf1 inactivation.

None of the osteocyte specific genes, with exception of Phex that is expressed both in osteocytes and osteoblasts, were significantly deregulated in Nf1Prx1 cortical bone tissue. This suggest that observed changes might in fact mostly be related to the defective osteoblastosteocyte transition rather than a primary osteocyte dysfunction. In support of these observations, many osteocytes appeared immersed in the immature collagen which was deposited within the perilacunar space (TEM). This phenotype is reminiscent of excessive collagen synthesis by osteoblasts and is paralleled by a global, likely compensatory, transcriptional downregulation of matrix gene expression. We also think that transcriptional upregulation of Phex, a gene known to counteract osteoidosis in Hyp mice, might be a compensatory effect [34]. Normal levels of the mineralization regulating factor Fgf23 in Nf1Prx1 plasma strengthen the idea that osteocytes are not the primary cause of the defect. Changes in osteocytes rather reflect the end stage of perturbed osteoblast differentiation. Thus, while Nf1Prx1 mice demonstrate an osteomalatic phenotype the overall phenotype is clearly distinct from the osteomalacia found in mice bearing Fgf23, Dmp1 and Phex gene inactivation $[9,35]$. The fact that we did not detect empty osteocyte lacunae (lacunae contained nuclei) demonstrated normal osteocyte viability. This was confirmed by negative results of the TUNEL assay and a normal PARP and LCII cleavage status. Increased microporosity due to enlarged osteocyte lacuna size contributes to deficiency of mechanical resistance in Nf1Prx1 bone tissue [16]. Unexpectedly, increased osteocyte size is likely attributed to improper cellular differentiation rather than a lacuna mineralization defect (normal mineral gradient around osteocyte lacunae). This conclusion is in line with unaffected Fgf23, Dmp1 and Phex levels in Nf1Prx1 bone tissue. Hyperosteoidosis in NF1 is likely due to imbalanced organic matrix maturation and subsequent mineralization $[13,14,16,29]$. However, very large mineralization lesions within Nf1Prx1 long bone diaphysis are likely caused by an interplay of persistent blood vessels, improper bone matrix quality and high mechanical loads [16]. The deltoid tuberosity in Nf1Prx1 and to a lesser degree in Nf1Col1 humerus is malformed [16]. This suggests that the site-specific effects at the ligament to bone insertion site, affecting chondrocyte and osteoblast development, might impact periosteal function rather than mechanical loading itself. If osteocytes act as a local amplifier of these effects awaits further analysis.

Loss of $N f 1$ in mesenchymal progenitor cells (Nf1Prx1 mice) produces a complex phenotype resulting in deficient long bone growth, tubular bone bowing, hip joint fusion, defective growth plate function, blood vessels persistence within the bone cortex, and muscle dystrophy $[14,16,17]$. A much milder bone phenotype, mainly characterized by hyperosteoidosis and high bone turnover, is observed in Nf1Col1 mice in which ablation of $N f 1$ is restricted to bone forming cells and occurs at the pre-osteoblast stage [13]. Our current view of the NF1 bone pathology, based on the analysis of growth plate chondrocyte, osteoblast and osteoclast dysfunction, is that increased bone turnover and an imbalance between extracellular matrix (ECM) synthesis and mineralization cause the NF1 skeletal manifestations [13,14,16,17,29,30]. Recently, in depth structural analysis of Nf1Prx1 mice suggested a critical role of the blood vessel system for the full NF1 pathology [16]. Here we demonstrate that Nf1 ablation has a developmental impact also on osteocytes. Importantly, morphological alterations of osteocytes are more pronounced in Nf1Prx1 rather than Nf1Col1 bone tissue. Thus NF1 inactivation in mesenchymal progenitor cells, affecting mesenchymal tissue development, $a b$ initio causes more pronounce changes to osteocyte development then the inactivation later in osteoblasts. This is likely due to a vital role which $N f 1$ plays in early osteoblast lineage differentiation and/or influence of other mesenchymal tissues including blood vessels and muscles [13,14,17,36].

Some aspects of our molecular analysis likely relate to the increased presence of blood vessels in the cortical bone of Nf1Prx1 mice [16]. A possible hint towards the molecular mechanism of the blood vessel associated bone lesions is the upregulation of Mgp in Nf1Prx1 bones. Mgp is a potent mineralization inhibitor expressed by osteoblasts and endothelial cells and its expression is regulated by phosphate in a MAPK dependent manner [37]. The involvement of Mgp in Nf1Prx1 cortical bone pathogenesis seems likely as Mgp knock-out causes vessel calcification and Mgp overexpression in osteoblasts results in hyperosteoidosis similar to the one observed in Nf1Prx1 mice [38]. It appears also likely that changes in the properties of $N f 1$ deficient vessels themselves contribute to defective bone mineralization. In support of this, Tem7/Plxdc1, a tumour endothelial marker expressed also by osteosarcoma cells, was among 
the upregulated genes in $N f 1$ deficient bones [39]. Increased expression of Tem7/Plxdc1 suggests that blood vessels in Nf1Prx1 bones have tumorlike properties [40] and might develop altered blood vessel barrier and diffusion properties [41]. This hypothesis awaits future experimental verification. Importantly, this study reveals that osteocytes, which are the most abundant cell type within cortical bone, are critically affected by the loss of neurofibromin. Morphological features as well as gene expression changes indicate perturbed cytoskeletal function, changes in energetic metabolism and altered extracellular matrix properties. We conclude that neurofibromin is required for mesenchymal lineage development and its inactivation hinders normal osteocyte development.

\section{Conflict of interest}

Authors have no competing interests to declare.

\section{Acknowledgments}

We thank Petra Schrade and Sebastian Bachmann from the electron microscopy core facility of the Charité - Universitätsmedizin Berlin for excellent assistance. We also thank Monika Osswald for excellent technical assistance.

Authors' roles: Study design: JK, FE, PF, SM, MK. Performed research: JK, JS, CL, SS, KK, JG, MK. Analyzed data: JK, MK. Wrote the manuscript: $\mathrm{JK}, \mathrm{MK}$. All authors read and approved the manuscript.

\section{Appendix A. Supplementary data}

Supplementary data to this article can be found online at http://dx. doi.org/10.1016/j.bone.2014.06.012.

\section{References}

[1] Bonewald LF. The amazing osteocyte. J Bone Miner Res 2011;26:229-38.

[2] Zhao S, Zhang YK, Harris S, Ahuja SS, Bonewald LF. MLO-Y4 osteocyte-like cells support osteoclast formation and activation. J Bone Miner Res 2002;17:2068-79.

[3] Nakashima T, Hayashi M, Fukunaga T, Kurata K, Oh-Hora M, Feng JQ et al. Evidence for osteocyte regulation of bone homeostasis through RANKL expression. Nat Med 2011;17:1231-4.

[4] Thi MM, Suadicani SO, Schaffler MB, Weinbaum S, Spray DC. Mechanosensory responses of osteocytes to physiological forces occur along processes and not cell body and require alphaVbeta3 integrin. Proc Natl Acad Sci U S A 2013;110:21012-7.

[5] Galli C, Passeri G, Macaluso GM. Osteocytes and WNT: the mechanical control of bone formation. J Dent Res 2010;89:331-43.

[6] Holmbeck K, Bianco P, Pidoux I, Inoue S, Billinghurst RC, Wu W, et al. The metalloproteinase MT1-MMP is required for normal development and maintenance of osteocyte processes in bone. J Cell Sci 2005;118:147-56.

[7] Khoshniat S, Bourgine A, Julien M, Petit M, Pilet P, Rouillon T, et al. Phosphatedependent stimulation of MGP and OPN expression in osteoblasts via the ERK1/2 pathway is modulated by calcium. Bone 2011;48:894-902.

[8] Feng JQ Ward LM, Liu S, Lu Y, Xie Y, Yuan B, et al. Loss of DMP1 causes rickets and osteomalacia and identifies a role for osteocytes in mineral metabolism. Nat Genet 2006;38:1310-5.

[9] Martin A, Liu S, David V, Li H, Karydis A, Feng JQ et al. Bone proteins PHEX and DMP1 regulate fibroblastic growth factor Fgf23 expression in osteocytes through a common pathway involving FGF receptor (FGFR) signaling. FASEB J 2011;25:2551-62.

[10] Plotkin LI, Mathov I, Aguirre JI, Parfitt AM, Manolagas SC, Bellido T. Mechanical stimulation prevents osteocyte apoptosis: requirement of integrins, Src kinases, and ERKs. Am J Physiol Cell Physiol 2005;289:C633-43.

[11] Kyono A, Avishai N, Ouyang Z, Landreth GE, Murakami S. FGF and ERK signaling coordinately regulate mineralization-related genes and play essential roles in osteocyte differentiation. J Bone Miner Metab 2012;30:19-30.

[12] Xia X, Kar R, Gluhak-Heinrich J, Yao W, Lane NE, Bonewald LF, et al. Glucocorticoidinduced autophagy in osteocytes. J Bone Miner Res 2010;25:2479-88.

[13] Elefteriou F, Benson MD, Sowa H, Starbuck M, Liu X, Ron D, et al. ATF4 mediation of NF1 functions in osteoblast reveals a nutritional basis for congenital skeletal dysplasiae. Cell Metab 2006;4:441-51.
[14] Kolanczyk M, Kossler N, Kuhnisch J, Lavitas L, Stricker S, Wilkening U, et al. Multiple roles for neurofibromin in skeletal development and growth. Hum Mol Genet 2007; 16:874-86.

[15] Stevenson DA, Zhou H, Ashrafi S, Messiaen LM, Carey JC, D'Astous JL, et al. Double inactivation of NF1 in tibial pseudarthrosis. Am J Hum Genet 2006;79:143-8.

[16] Kuhnisch J, Seto J, Lange C, Schrof S, Stumpp S, Kobus K, et al. Multiscale, Converging Defects of Macro-Porosity, Microstructure and Matrix Mineralization Impact Long Bone Fragility in NF1. PLoS One 2014;9:e86115.

[17] Kossler N, Stricker S, Rodelsperger C, Robinson PN, Kim J, Dietrich C, et al Neurofibromin (Nf1) is required for skeletal muscle development. Hum Mol Genet 2011;20:2697-709.

[18] Kolanczyk M, Kuhnisch J, Kossler N, Osswald M, Stumpp S, Thurisch B, et al Modelling neurofibromatosis type 1 tibial dysplasia and its treatment with lovastatin. BMC Med 2008;6:21.

[19] Morava E, Kuhnisch J, Drijvers JM, Robben JH, Cremers C, van Setten P, et al. Autosomal recessive mental retardation, deafness, ankylosis, and mild hypophosphatemia associated with a novel ANKH mutation in a consanguineous family. J Clin Endocrinol Metab 2011;96:E189-98

[20] Pascaretti-Grizon F, Gaudin-Audrain C, Gallois Y, Retaillaud-Gaborit N, Basle MF, Chappard D. Osteopontin is an argentophilic protein in the bone matrix and in cells of kidney convoluted tubules. Morphologie 2007;91:180-5.

[21] Dayan D, Hiss Y, Hirshberg A, Bubis JJ, Wolman M. Are the polarization colors of picrosirius red-stained collagen determined only by the diameter of the fibers? Histochemistry 1989;93:27-9.

[22] Parfitt AM, Drezner MK, Glorieux FH, Kanis JA, Malluche H, Meunier PJ, et al. Bone histomorphometry: standardization of nomenclature, symbols, and units. Report of the ASBMR Histomorphometry Nomenclature Committee. J Bone Miner Res 1987;2:595-610.

[23] Kawamoto T, Shimizu M. A method for preparing 2- to 50-micron-thick fresh-frozen sections of large samples and undecalcified hard tissues. Histochem Cell Biol 2000;113:331-9.

[24] Roschger P, Fratzl P, Eschberger J, Klaushofer K. Validation of quantitative backscattered electron imaging for the measurement of mineral density distribution in human bone biopsies. Bone 1998;23:319-26.

[25] Cully M, Downward J. SnapShot: Ras Signaling. Cell 2008;133:1292-1292 [e1].

[26] Xu J, Wang S, Viollet B, Zou MH. Regulation of the proteasome by AMPK in endothelial cells: the role of O-GlcNAc transferase (OGT). PLoS One 2012;7:e36717.

[27] Rowe PS. The chicken or the egg: PHEX, FGF23 and SIBLINGs unscrambled. Cell Biochem Funct 2012;30:355-75.

[28] Huang da W, Sherman BT, Lempicki RA. Systematic and integrative analysis of large gene lists using DAVID bioinformatics resources. Nat Protoc 2009;4:44-57.

[29] Seitz S, Schnabel C, Busse B, Schmidt HU, Beil FT, Friedrich RE, et al. High bone turnover and accumulation of osteoid in patients with neurofibromatosis 1. Osteoporos Int 2010;21:119-27.

[30] Lammert M, Kappler M, Mautner VF, Lammert K, Storkel S, Friedman JM, et al. Decreased bone mineral density in patients with neurofibromatosis 1 . Osteoporos Int 2005; 16:1161-6.

[31] Ozawa T, Araki N, Yunoue S, Tokuo H, Feng L, Patrakitkomjorn S, et al. The neurofibromatosis type 1 gene product neurofibromin enhances cell motility by regulating actin filament dynamics via the Rho-ROCK-LIMK2-cofilin pathway. J Biol Chem 2005;280:39524-33.

[32] Starinsky-Elbaz S, Faigenbloom L, Friedman E, Stein R, Kloog Y. The pre-GAP-related domain of neurofibromin regulates cell migration through the LIM kinase/cofilin pathway. Mol Cell Neurosci 2009;42:278-87.

[33] Oakhill JS, Steel R, Chen ZP, Scott JW, Ling N, Tam S, et al. AMPK is a direct adenylate charge-regulated protein kinase. Science 2011;332:1433-5.

[34] Erben RG, Mayer D, Weber K, Jonsson K, Juppner H, Lanske B. Overexpression of human PHEX under the human beta-actin promoter does not fully rescue the Hyp mouse phenotype. J Bone Miner Res 2005;20:1149-60.

[35] Shimada T, Kakitani M, Yamazaki Y, Hasegawa H, Takeuchi Y, Fujita T, et al. Targeted ablation of Fgf23 demonstrates an essential physiological role of FGF23 in phosphate and vitamin D metabolism. J Clin Invest 2004;113:561-8.

[36] Ono K, Karolak MR, Ndong JD, Wang W, Yang X, Elefteriou F. The Ras-GTPase activity of neurofibromin restrains ERK-dependent FGFR signaling during endochondral bone formation. Hum Mol Genet Aug. 1 2013;22(15):3048-62.

[37] Julien M, Khoshniat S, Lacreusette A, Gatius M, Bozec A, Wagner EF, et al. Phosphatedependent regulation of MGP in osteoblasts: role of ERK1/2 and Fra-1. J Bone Miner Res 2009;24:1856-68.

[38] Murshed M, Schinke T, McKee MD, Karsenty G. Extracellular matrix mineralization is regulated locally; different roles of two gla-containing proteins. J Cell Biol 2004;165:625-30

[39] Halder C, Ossendorf C, Maran A, Yaszemski M, Bolander ME, Fuchs B, et al. Preferential expression of the secreted and membrane forms of tumor endothelial marker 7 transcripts in osteosarcoma. Anticancer Res 2009;29:4317-22.

[40] van Beijnum JR, Petersen K, Griffioen AW. Tumor endothelium is characterized by a matrix remodeling signature. Front Biosci (Schol Ed) 2009;1:216-25.

[41] Dudley AC. Tumor endothelial cells. Cold Spring Harb Perspect Med 2012;2: a006536. 